\title{
Distúrbios evacuatórios em primigestas após parto normal: estudo clínico
}

\author{
Evacuatory disorders in primigravidae after vaginal delivery: clinical study
}

\author{
MARIA AUXILIADORA PROLUNGATTI CESAR ${ }^{1}$, JÚLIA MOTA LEITE², RAFAELA CRISTINA COELHO MUNIZ², \\ JORGE ALBERTO ORTIZ ${ }^{3}$
}

\begin{abstract}
${ }^{1}$ Mestre e Doutora em Medicina pela Faculdade de Ciências Médicas da Santa Casa de São Paulo; Professor Assistente Doutor da Disciplina de Cirurgia do Departamento de Medicina da Universidade de Taubaté (UNITAU) e Responsável pelo Ambulatório de Fisiologia Anal do Hospital Universitário de Taubaté - Taubaté (SP), Brasil. ${ }^{2}$ Médica formada pela UNITAU - Taubaté (SP), Brasil. ${ }^{3}$ Mestre em Medicina pela Faculdade de Ciências Médicas da Santa Casa de São Paulo-
\end{abstract} São Paulo (SP), Brasil.

CESAR MAP, LEITE JM, MUNIZ RCC, ORTIZ JA. Distúrbios evacuatórios em primigestas após parto normal: estudo clínico. Rev bras Coloproct, 2011;31(2): 126-130.

RESUMO: Introdução: Os distúrbios evacuatórios são descritos na literatura relacionados ao parto normal e episiotomia. Objetivo: Estudar a incidência de distúrbios evacuatórios em primíparas submetidas à episiotomia, durante o parto normal. Métodos: Estudo prospectivo em 32 pacientes primíparas, submetidas à episotomia durante parto vaginal. Para realização do estudo as pacientes responderam os questionários com o score de constipação de Agachan e score de incontinência de Jorge e Wexner, em relação ao período pré-gestacional e 90 dias após o parto. Resultados: Não foram encontradas diferenças estatisticamente significantes quando comparados os resultados dos questionários aplicados, em relação ao período pré-gestacional e 90 dias pós-parto. Conclusões: Não observamos alterações da constipação e continência fecal em primigestas após parto normal com episiotomia

Palavras-chave: parto normal; constipação intestinal; incontinência fecal; episiotomia; gestação.

\section{INTRODUÇÃO}

Apesar de o parto normal ser considerado fisiológico e mais adequado, existe relatos sobre a frequência e consequências do traumatismo anal de causa obstétrica, sendo causas comuns dos distúrbios evacuatórios no pós-parto normal ${ }^{1-3}$.

Dentre os distúrbios, a incontinência anal pode ser definida como a incapacidade de manter o controle fisiológico do conteúdo intestinal, em local e tempo socialmente adequados. É caracterizada pela perda involuntária de fezes sólidas e líquidas associada ao escape ocasional de flatos ${ }^{4,5}$.

Estudos demonstram que a "manipulação vaginal" e a episiotomia, realizados durante o parto vagi- nal, são fatores de risco para disfunções do diafragma pélvico no pós-parto. Trauma direto ao esfíncter anal e neuropatias do nervo pudendo são os dois eventos mais comuns que ocorrem durante o parto e explicam o desenvolvimento de incontinência fecal, após o mesmo ${ }^{3,5-8}$.

Outro distúrbio evacuatório frequente é a constipação intestinal, que é definida como a presença de menos de três evacuações por semana, ou a presença de evacuação com dificuldade, ou esforço, ou incompleta ${ }^{9-11}$.

Para a avaliação e classificação dos distúrbios evacuatórios, podem ser utilizados questionários como o escore de constipação de Agachan $^{12}$ e o escore de incontinência de Jorge e Wexner ${ }^{13,14}$.

Trabalho realizado nos Serviços de Clínica Cirúrgica e Ginecologia e Obstetrícia do Hospital Universitário de Taubaté - Taubaté (SP), Brasil.

Fonte de financiamento: não há

Conflito de interesse: nada a declarar

Recebido em: $28 / 02 / 2011$

Aprovado em: 25/03/2011 
O objetivo deste trabalho foi avaliar a incidência de constipação e incontinência fecal após parto normal com episiotomia em primíparas.

\section{MÉTODO}

Foi realizado um estudo prospectivo, com objetivo de avaliar a incidência de constipação e incontinência anal após parto normal em primíparas, através da definição de escores de constipação de Agachan e de incontinência de Jorge e Wexner.

Os critérios de inclusão foram: primíparas, pacientes da enfermaria de obstetrícia e que se submeteram ao parto normal no período de setembro a dezembro de 2009, parto a partir de 36 semanas. Os critérios de exclusão foram os seguintes: cirurgias anorretais prévias, presença de sintomas de incontinência fecal para fezes ou gases com escore de Jorge e Wexner maior que cinco, presença de sintomas de constipação intestinal com escore de Agachan maior que dez, parto cesárea, presença de doenças sistêmicas (diabetes, hipertireoidismo).

Todos os pacientes assinaram um termo de consentimento livre e esclarecido para participar do estudo.

Para realização do estudo as pacientes responderam questionário contendo o escore de constipação de Agachan e o escore de incontinência de Jorge e Wexner em duas etapas: período pré-gestacional e 90 dias após o parto. Os escores citados se encontram detalhados nos Quadros 1 e 2.

\section{RESULTADOS}

Foram estudadas 32 pacientes, cuja média das idades foi de 20,75 $\pm 5,49$ anos, variando entre 13 e 35 anos.

Em relação ao escore de Agachan, o valor mínimo foi zero no pré- e pós-parto, já o valor máximo variou de 10 no pré-parto a 17 no pós-parto.

Os valores obtidos utilizando-se o escore de Agachan encontram-se dispostos na Tabela 1 e não encontramos diferenças entre as duas etapas estudadas ( período pré-gestacional e 90 dias pós-parto).

Em relação ao escore de incontinência de Jorge e Wexner o escore máximo foi de três no período prégestacional e de cinco no $90^{\circ}$ dia pós-parto. Já o mínimo foi de zero no pré-parto e no $90^{\circ}$ dia pós-parto. Os dados estão dispostos na Tabela 2 e não apresentam diferenças estatisticamente significantes.

\section{DISCUSSÃO}

Existe uma discussão ampla na literatura relacionada ao efeito do parto normal na constipação e, principalmente, na continência anal.

Em relação à constipação intestinal relacionada ao parto, esperávamos encontrar algumas diferenças, principalmente relacionadas ao fato de as primíparas terem uma menor frequência evacuatória, relacionada aos cuidados com a criança, mas isso não foi encontrado.

Quadro 1. Escore de constipação de Agachan.

\begin{tabular}{lccccc}
$\begin{array}{l}\text { Tipo de } \\
\text { incontinência }\end{array}$ & Frequência & \multicolumn{5}{c}{ Escore } \\
\hline & Nunca & $\begin{array}{c}\text { Até uma } \\
\text { vez ao mês }\end{array}$ & $\begin{array}{c}\text { Entre uma vez por } \\
\text { semana e uma ou mais } \\
\text { vezes ao mês }\end{array}$ & $\begin{array}{c}\text { Entre uma vez ao dia e } \\
\text { uma ou mais vezes por } \\
\text { semana }\end{array}$ & $\begin{array}{c}\text { Mez de uma dia } \\
\text { vez ao }\end{array}$ \\
\hline Sólidos & 0 & 1 & 2 & 3 & 4 \\
Líquidos & 0 & 1 & 2 & 3 & 4 \\
Gases & 0 & 1 & 2 & 3 & 4 \\
$\begin{array}{l}\text { Uso de protetores } \\
\text { de roupa }\end{array}$ & 0 & 1 & 2 & 3 & 4 \\
$\begin{array}{l}\text { Alteração na } \\
\text { qualidade de vida }\end{array}$ & 0 & 1 & 2 & 3 & \\
Total & & & & & \\
\hline
\end{tabular}


Quadro 2. Escore de incontinência de Jorge e Wexner

\begin{tabular}{|c|c|c|c|c|c|}
\hline \multirow{2}{*}{ Escore de Constipação } & \multicolumn{5}{|c|}{ Escore } \\
\hline & $\mathbf{0}$ & 1 & 2 & 3 & 4 \\
\hline Frequência intestinal & $\begin{array}{l}1-2 \times \text { por dia } \\
\text { em } 1-2 \text { dias }\end{array}$ & $\begin{array}{l}2 \times \text { por } \\
\text { semana }\end{array}$ & $\begin{array}{l}1 \times \text { por } \\
\text { semana }\end{array}$ & $\begin{array}{l}\text { Menos de } 1 \mathrm{x} \\
\text { por semana }\end{array}$ & $\begin{array}{l}\text { Menos que } 1 \\
\mathrm{x} \text { por mês }\end{array}$ \\
\hline $\begin{array}{l}\text { Dificuldade: esforço evacuatório } \\
\text { doloroso }\end{array}$ & Nunca & Raramente & $\begin{array}{l}\text { Algumas } \\
\text { vezes }\end{array}$ & Geralmente & Sempre \\
\hline $\begin{array}{l}\text { Sensação de evacuação } \\
\text { incompleta }\end{array}$ & Nunca & Raramente & $\begin{array}{l}\text { Algumas } \\
\text { vezes }\end{array}$ & Geralmente & Sempre \\
\hline Dor abdominal & Nunca & Raramente & $\begin{array}{l}\text { Algumas } \\
\text { vezes }\end{array}$ & Geralmente & Sempre \\
\hline $\begin{array}{l}\text { Tempo: minutos no lavatório, } \\
\text { tentativa para evacuar }\end{array}$ & Menos que 5 & $5-10$ & $10-20$ & $20-30$ & Mais que 30 \\
\hline $\begin{array}{l}\text { Auxílio: tipo de auxílio para } \\
\text { evacuar }\end{array}$ & Sem auxílio & $\begin{array}{l}\text { Laxativos } \\
\text { estimulantes }\end{array}$ & $\begin{array}{l}\text { Auxílio } \\
\text { digital ou } \\
\text { enema }\end{array}$ & 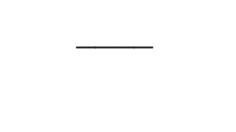 & - \\
\hline $\begin{array}{l}\text { Tentativa para evacuar sem } \\
\text { sucesso em } 24 \mathrm{~h} \text { (TESS em } 24 \mathrm{~h})\end{array}$ & Nunca & Menos que 3 & $3-6$ & $6-9$ & Maior que 9 \\
\hline Duração da constipação em anos & 0 & $1-5$ & $5-10$ & $10-20$ & Maior que 20 \\
\hline Total & & & & & \\
\hline
\end{tabular}

Variáveis analisadas pelo escore de Jorge e Wexner para avaliar o grau de constipação intestinal.

Tabela 1. Ritmo intestinal pré-gestacional e após parto normal em primigestas, segundo o escore de Agachan.

\begin{tabular}{lcccccccc}
\hline & Grupo & N & MEDIA & $\begin{array}{c}\text { Desvio } \\
\text { padrão }\end{array}$ & Mínima & Mediana & Máxima & MW \\
\hline Frequência & 1 & 32 & 0,37 & 0,65 & 0 & 0,00 & 3,00 & 0,61 \\
& 2 & & 0,37 & 0,75 & 0 & 0,00 & 2,00 & \\
Esforço doloroso & 1 & 32 & 0,96 & 1,14 & 0 & 1,00 & 4,00 & 0,19 \\
& 2 & 32 & 1,50 & 1,50 & 0 & 1,00 & 4,00 & \\
Evacuação incompleta & 1 & 32 & 0,62 & 0,79 & 0 & 0,00 & 2,00 & 0,35 \\
& 2 & 32 & 1,09 & 1,42 & 0 & 0,00 & 4,00 & \\
Dor abdominal & 1 & 32 & 0,59 & 0,83 & 0 & 0,00 & 3,00 & 0,29 \\
& 2 & 32 & 0,93 & 1,18 & 0 & 0,05 & 4,00 & \\
Tempo & 1 & 32 & 0,53 & 0,67 & 0 & 0,00 & 2,00 & 0,31 \\
& 2 & 32 & 0,34 & 0,48 & 0 & 0,00 & 1,00 & \\
Tentativa para evacuar & 1 & 32 & 0,62 & 0,75 & 0 & 0,00 & 3,00 & 0,40 \\
sem sucesso em 24h & 2 & 32 & 0,62 & 1,09 & 0 & 0,00 & 4,00 & \\
Tipo de auxílio & 1 & 32 & 0,12 & 0,33 & 0 & 0,00 & 1,00 & 0,72 \\
utilizado para evacuar & 2 & 32 & 0,15 & 0,36 & 0,00 & 0,00 & 1,00 & \\
Total & 1 & 32 & 4,28 & 2,63 & 0 & 4,00 & 10,00 & \\
& 2 & 32 & 5,37 & 5,04 & 0,00 & 4,00 & 17,00 & 0,94 \\
\hline
\end{tabular}

Grupo 1: período pré-gestacional; Grupo 2: 90 dias pós-parto; N: número de pacientes analisados; MW: Mann-Whitney. 
Tabela 2. Valores do escore de incontinência anal de acordo com o escore de Wexner em primíparas no período pré-gestacional e pós-parto.

\begin{tabular}{lcccccccc}
\hline Tipo de incontinência & Grupo & N & Média & $\begin{array}{c}\text { Desvio } \\
\text { padrão }\end{array}$ & Mínima & Mediana & Maxima & MW \\
\hline Sólidos & 1 & 32 & 0,00 & 0,00 & 0,00 & 0,00 & 0,00 & 0,00 \\
Líquidos & 2 & 32 & 0,00 & 0,00 & 0,00 & 0,00 & 0,00 & \\
& 1 & 32 & 0,12 & 0,42 & 0,00 & 0,00 & 2,00 & 0,07 \\
Gases & 2 & 32 & 0,00 & 0,00 & 0,00 & 0,00 & 0,00 & \\
& 1 & 32 & 0,28 & 0,72 & 0,00 & 0,00 & 3,00 & 0,32 \\
Uso de protetores de & 2 & 32 & 0,53 & 1,04 & 0,00 & 0,00 & 4,00 & \\
roupa & 1 & 32 & 0,00 & 0,00 & 0,00 & 0,00 & 0,00 & 0,00 \\
Alteração na qualidade & 2 & 32 & 0,00 & 0,00 & 0,00 & 0,00 & 0,00 & \\
de vida & 1 & 32 & 0,12 & 0,42 & 0,00 & 0,00 & 2,00 & 0,96 \\
Total & 2 & 32 & 0,09 & 0,29 & 0,00 & 0,00 & 1,00 & \\
& 1 & 32 & 1,53 & 1,04 & 1,00 & 1,00 & 4.00 & 0,00 \\
\hline
\end{tabular}

Grupo 1: Periodo pré-gestacional; Grupo 2: 90 dias pós-parto; N: número de pacientes analisados; DP: Desvio padrão; MW: MannWhitney.

Uma demora para atender a sensação retal pode ser a causa de constipação; quando a paciente não atende a primeira vontade de evacuar, pode ocorrer uma absorção de água das fezes, ocasionando fezes ressecadas e, consequentemente, maior tempo evacuatório, necessidade de esforço evacuatório, evacuação dolorosa ${ }^{1,2,8-11}$.

Observamos que, no grupo após o parto, existe uma maior tendência a distúrbios evacuatórios, relacionados a maior tempo evacuatório e esforço intestinal doloroso, mas isso não se refletiu nos dados estatísticos.

O parto vaginal apresenta maior incidência para o desenvolvimento de incontinência fecal no pós-parto em relação à cesárea eletiva; porém, a mesma não protege contra o aparecimento dessa patologia. O que justifica contraindicação de cesárea eletiva para prevenção de incontinência fecal pós-parto ${ }^{6}$.

Estudos mostram que mulheres apresentam maior risco para a incontinência fecal, considerandose como fatores de risco as lesões do nervo pudendo ou do esfíncter anal por trauma obstétrico, lesão cirúrgica do esfíncter anal, distopias genitais e neuropatia diabética ${ }^{5}$.

Podemos, ainda, citar outros fatores de risco, como o uso de fórceps, cesáreas de emergência, analgesia epidural, laceração perineal, fístula obstétrica genital, tempo prolongado da segunda fase do trabalho de parto ${ }^{3,6,7}$.

Não encontramos em nossa casuística alterações significativas na continência provocadas pelo parto normal, o que provavelmente reflete a realização de episiotomias com técnica adequada, não causando lesões esfincterianas e, consequentemente, distúrbios da continência. Para estas pacientes, seria interessante, para uma avaliação mais precisa da ausência de lesão esfincteriana, a realização de manometria anal e/ ou ultrassonografia retal, o que estava proposto em nossa pesquisa, mas não obtivemos adesão das mesmas para a realização, tanto no período pré-, quanto no pós-parto.

\section{CONCLUSÃO}

Concluímos que o parto normal com episiotomia em primigestas não causou distúrbios de continência fecal e constipação no pós-parto em nossa casuística.

\section{AGRADECIMENTOS}

Aos doutores Pedro Roberto de Paula e Renato Coimbra Mazzini pelas sugestões na confecção desta pesquisa. 
ABSTRACT: Introduction: Evacuatories disturbances are described in the literature related to vaginal delivery and episiotomy. Objective: To study the incidence of evacuatories disturbances in primiparous undergoing episiotomy during vaginal delivery. Methods: Prospective study in 32 primiparous patients submitted to episotomy during vaginal delivery. For the study the patients answered in relation to the period before pregnancy and 90 days postpartum questionnaires with the score of Agachan constipation and incontinence score of Jorge and Wexner. Results: No statistically significant differences were found when comparing the results from the questionnaires in relation to the period before pregnancy and 90 days postpartum. Conclusions: Episiotomy in vaginal delivery in primiparous women with no prior history of intestinal symptoms, caused no disorders of anal continence postpartum in the pacients of this study.

Keywords: natural childbirth; constipation; incontinence; episiotomy; pregnancy.

\section{REFERÊNCIAS}

1. Klug WA, Aguida HAC, Ortiz JA, Fang CB, Capelhuchnik P. Alterações das pressões anais na gravidez. Rev bras Coloproct 2007;27(2):196-201.

2. Klug WA, Aguida HAC, Ortiz JA, Fang CB, Capelhuchnik P. Pressões retais e anais em primigestas ao defecar. Rev Assoc Med Bras 2008;54(2):150-3.

3. Rogers RG, Leeman LM, Miglioccio L, Albers LL. Does the severity of spontaneous genital tract trauma affect postpartum pelvic floor fuction? Int Urogynecol J Pelvic Floor Dysfunct 2008;19(3):429-35.

4. Carvalho LP, Corleta OC, Mallmann ACM, Koshimizu RT, Spolavori A. Neuropatia pudenda: correlação com dados demográficos, índice de gravidade e parâmetros pressóricos em pacientes com incontinência fecal. Arq Gatroenterol 2002;39(3):139-46.

5. Oliveira SCM, Pinto-Neto AM, Conde DM, Góes JRN, Santos-Sá D, Costa-Paiva L. Incontinência fecal em mulheres na pós-menopausa: prevalência, intensidade e fatores associados. Arq Gastroenterol 2006;43(2):102-6.

6. Quiegley EMM. Impact of pregnancy and parturitiun on the anal sphincter and pelvic floor. Best Pract Res Cil Gastroenterol 2007;21(5):879-91.

7. Groutz A, Fait G, Lessing JB, David MP, Wolman I, Jaffa A, et al. Incidence and obstetric risk factors of postpartum anal incontinence. Scand J Gastroenterol 1999;34(3):315-8.

8. Nazir M, Carlsen E, Nesheim BI. Do occult anal sphincter injuries, vector volume manometry and delivery variables have any predictive value for bowel symptoms after firts time vaginal delivery without third and fourth degree rupture? A prospecty study. Acta Obstet Gynecol Scand 2002;81(8):720-6.

9. Kawaguti FS, Klug WA, Fang CB, Ortiz JA, Capelhucnick P. Constipação na gravidez. Rev bras Coloproct 2008;28(1):46-9.

10. César MAP, Klug WA, Aguida HAC, Ortiz JA, Fang CB, Capelhuchnik P. Alterações das pressões anais em pacientes constipados por defecação obstruída. Rev bras Coloproct 2008;28(4):402-8.

11. Lacerda-Filho AF, Lima MJR, Magalhães MF, Paiva RA, Cunha-Melo JR. O papel da avaliação clínica e dos testes de fisiologia colo-reto anal no diagnóstico etiológico da constipação intestinal crônica. Arq Gastrenterol 2008;45(1):50-7.

12. Agachan $\mathrm{F}$, Chen $\mathrm{T}$, Pfeifer $\mathrm{J}$, Reissman P, Wexner SD. A constipation scoring system to simplify evaluation and management of constipated patients. Dis Colon Rectum 1996;39(6):681-5.

13. Jorge JM, Wexner SD. Etiology and management of fecal incontinence. Dis Colon Rectum 1993;36(1):77-97.

14. Yusuf SAI, Jorge JMN, Habr-Gama A, Kiss DR, Gama Rodrigues J. Avaliação da qualidade de vida na incontinência anal: validação do questionário FIQL (Fecal Incontinence Quality Of Life). Arq Gastroenterl 2004;41(3):202-8.

Endereço para correspondência:

Maria Auxiliadora Prolungatti Cesar

Serviço de Clínica Cirúrgica do Hospital Universitário de Taubaté Avenida Granadeiro Guimarães, 270

CEP: 12020-130 - Taubaté (SP), Brasil.

E mail: prolungatti@uol.com.br 